\title{
MAPEO MICROBIOLÓGICO DE Salmonella spp. EN PLANTAS DE DESPOSTE Y COMERCIALIZACIÓN
}

\section{MAPEO MICROBIOLÓGICO DE Salmonella spp. EN PLANTAS DE DESPOSTE Y COMERCIALIZACIÓN}

\author{
${ }^{1}$ Rojas C. ${ }^{*},{ }^{1}$ Fajardo M, ${ }^{1}$ Carrascal A \\ *1Pontificia Universidad Javeriana/Facultad de Ciencias/Departamento de Microbiología/Grupo de Biotecnología \\ Ambiental e Industrial/Semillero de Inocuidad Alimentaria/Bogotá/Colombia. \\ Correo electronico: carolrojas@javeriana.edu.co, maira-fajardo@javeriana.edu.co, acarrasc@javeriana.edu.co
}

Recibido. 21 de septiembre de 2017; Aceptado: 15 de diciembre de 2017.

\section{RESUMEN}

Las enfermedades transmitidas por alimentos (ETA) se han convertido en el problema en salud pública más extendido en el mundo. Según la OMS, el $95 \%$ de las ETA representan enfermedades diarreicas donde Salmonella es uno de los principales agentes causales. La presencia de este patógeno en la carne de cerdo se ha descrito como una fuente de infección al humano poniendo en riesgo su salud, si no se cuenta con programas de control y vigilancia durante la cadena de producción. Por lo cual, el objetivo de este estudio fue determinar la concentración de Salmonella spp. en plantas de desposte y comercialización en dos industrias porcinas de Colombia. Se realizó un muestreo por mes durante cinco 
Autor a quien dirigirse la correspondencia: Rojas C.*, Correo electronico:

carolrojas@javeriana.edu.co meses en cada empresa para un total de 425 muestras en plantas de desposte y comercialización, las cuales se analizaron mediante el método de Número Más Probable (NMP). La mayor concentración de Salmonella spp. se obtuvo en la etapa de comercialización en la industria $A$ y $B$ con valores de 4,252 NMP/g y 149,148 NMP/g respectivamente. Las altas concentraciones de Salmonella spp. en plantas de desposte y comercialización en la industria $B$ se debe principalmente a cerdos contaminados desde granja, contaminación cruzada durante el proceso y perdida de cadena de frío; por lo tanto, la implementación de sistemas preventivos ayudan a reducir y mantener bajo control la presencia del patógeno.

Palabras Claves: Comercialización, Número Más Probable, Mapeo microbiológico, Planta de desposte, Salmonella spp.

\section{ABSTRACT}

Foodborne illness have become in the most extended health public issue around the world. The World Health Organisation (WHO), shows that $95 \%$ of diarreic illnesses are foodborn illnesses whereSalmonella is a cauding agent. The presence of this pathogen in pork meat has been describes as a source of human infection that can threaten its health if there are not control and surveillance programs during production lines. That is why the goal of this paper was to determinate the concentration of Salmonella spp. in cutting plants and commercialization in two pork industries in Colombia. A sampling was made every month for five months in each pork industry for a total of 425 samples in cutting plants and commercialization stage, which were analyzed by the Most Probable Number method (MPN). The highest Salmonella concentration was obtained in the commercialization stage in both industries with 4,252 NMP/g in the A industry and 149,148 
$\mathrm{NMP} / \mathrm{g}$ in the B industry. High concentrations of Salmonella spp. in cutting plants and commercialization stage in the $B$ industry are mainly because pigs contaminated from the farms, crossed contamination during the process and loss of the cold chain; thus, the implementation of preventive systems help to reduce and maintain control of the pathogen.

Key Words: Commercialization, Most Probable Number, Microbiological mapping, Cutting plant, Salmonella spp.

\section{INTRODUCCIÓN}

Las enfermedades transmitidas por alimentos (ETA) son el problema en salud pública más extendido en el mundo, en la región de las Américas según la Organización Mundial de la Salud (OMS), causan al año 77 millones de personas enfermas de las cuales más de nueve mil mueren (OMS, 2015). El 95 \% de las ETA representan enfermedades diarreicas donde Salmonella es uno de los cuatro principales agentes causales (OMS, 2017). En Colombia en el año 2014, se registraron 11425 casos de ETA, donde Salmonella se asoció a brotes e identificaron como los principales factores de riesgo la contaminación cruzada y fallas en la cadena de frío (Guerrero, 2016 ; Pua, et al., 2014).

Salmonella spp. es un bacilo Gram negativo, anaerobio facultativo, crece en condiciones óptimas de temperatura entre $35-37^{\circ} \mathrm{C}$ y de $\mathrm{pH}$ entre 6.5 - 7.5. Este microorganismo se encuentra presente en el intestino de pollos y cerdos, sus principales reservorios (INS, 2011). Una de las enfermedades causada por esta bacteria, es salmonelosis la cual es una gastroenteritis que se caracteriza por causar diarrea, dolor abdominal, fiebre y dolor de cabeza. La duración media de la enfermedad es de aproximadamente 7 días, aunque esto depende de la edad, estado inmunológico de la persona y la concentración del microorganismo ingerida (Parra et al., 2002; INS, 2011).

Salmonella se puede encontrar en el intestino, faringe, ganglios mesentéricos y heces del cerdo, siendo reportada como una fuente de contaminación en la industria desde la producción primaria, generando un problema a la industria porcina, si los operarios realizan manejos inadecuados de la canal, haciendo que la bacteria atraviese 
toda la cadena de producción hasta llegar a los hogares, establecimientos o institutos de servicios de comida (OMS, 2017). Por esta razón es importante tener en cuenta que intervenciones en la producción primaria como la implementación de buenas prácticas porcícolas, ayuda a disminuir en primera instancia los patógenos presentes en el animal haciendo que la eliminación o disminución de las bacterias patógenas durante las siguientes etapas sea más fácil (Berends et al., 1996; Rodríguez, 2014).

El control de este patógeno ha tenido un gran impacto en la industria porcina de Colombia debido al crecimiento en el consumo de carne en el país el cual ascendió a 9.3 kilogramos por habitante (PorkColombia, 2018). Por este motivo las empresas productoras de carne de cerdo deben cumplir con el Decreto 1500 de 2007 del Ministerio de Protección Social para la inspección, vigilancia y control de la carne, productos y derivados cárnicos destinados al consumo humano, donde se establece el cumplimiento de los requisitos sanitarios y de inocuidad en la cadena de producción.

Por esta razón, se realizó este estudio el cual se basó en un mapeo microbiológico, donde el objetivo es cuantificar la concentración de Salmonella spp. en las de planta de desposte y comercialización, para poder establecer cual presenta mayor contaminación y estimar si las medidas tomadas por parte de las empresas en las diferentes etapas son las adecuada

\section{MATERIALES Y MÉTODOS}

El estudio se llevó acabo en dos industrias porcinas ( $\mathrm{A}$ y $\mathrm{B}$ ) escogidas a conveniencia, las cuales cuentan con la cadena de producción completa y participaron de manera voluntaria.

El muestreo se realizó una vez al mes durante cinco meses seguidos en las plantas de desposte y en los puntos de comercialización de las empresas, se tomaron un total de 425 muestras. En cada una de las etapas seleccionadas (ver tabla 1) se tomaron 5 muestras del mismo lote.

Las muestras fueron tomadas por un médico veterinario previamente entrenado, por medio del método de esponja y cortes de carne el cual siguió el siguiente plan de muestreo (ver tabla 1) (Rodríguez \& Carrascal). En la empresa $A$ no se realizó muestreo en la etapa de descargue, al no existir esta, ya que las canales se 
transportan del beneficio al desposte por vía aérea, al estar dentro la misma planta de producción.

Tabla 1. Plan de muestreo en planta.

\begin{tabular}{|c|c|c|c|}
\hline Lugar & Etapa & $\begin{array}{l}\text { Número de } \\
\text { muestras }\end{array}$ & $\begin{array}{l}\text { Toma de } \\
\text { muestra }\end{array}$ \\
\hline \multirow{4}{*}{$\begin{array}{l}\text { Planta } \\
\text { de } \\
\text { despos } \\
\text { te }\end{array}$} & Descargue & 5 & $\begin{array}{l}\text { Método } \\
\text { de } \\
\text { esponja }\end{array}$ \\
\hline & Cuarto frío & 5 & $\begin{array}{l}\text { Método } \\
\text { de } \\
\text { esponja }\end{array}$ \\
\hline & $\begin{array}{l}\text { Cuarto } \\
\text { desposte }\end{array}$ & 5 & $\begin{array}{l}100 \\
\text { gramos } \\
\text { de la } \\
\text { canal }\end{array}$ \\
\hline & $\begin{array}{l}\text { Cortes de } \\
\text { carne }\end{array}$ & 15 & $\begin{array}{l}100 \\
\text { gramos } \\
\text { por cada } \\
\text { corte de } \\
\text { carne } \\
\text { (chuleta, } \\
\text { brazo, } \\
\text { lomo) }\end{array}$ \\
\hline $\begin{array}{c}\text { Comerc } \\
\text { ializaci } \\
\text { ón }\end{array}$ & $\begin{array}{l}\text { Punto de } \\
\text { venta }\end{array}$ & 15 & $\begin{array}{l}50 \\
\text { gramos } \\
\text { por cada } \\
\text { corte de } \\
\text { carne } \\
\text { (chuleta, } \\
\text { brazo, } \\
\text { lomo) }\end{array}$ \\
\hline
\end{tabular}

Las muestras se conservaron bajo condiciones de refrigeración (inferior a $4^{\circ} \mathrm{C}$ ) en neveras portátiles, el procesamiento se realizó en el Laboratorio de Microbiología de Alimentos de la Pontificia Universidad Javeriana.

\section{- $\quad$ Método de Número Más Probable}

Para el análisis de las muestras y cuantificación de Salmonella se adaptó el método NMP miniaturizado descrito por Pavic et al. (2010). Primero se pesaron 10 gramos de la carne y se añadieron en $90 \mathrm{ml}$ de agua peptonada bufferada y se llevó al
Stomacher por 30 segundos, después se realizaron diluciones seriadas hasta $10^{-3}$, a partir de las diluciones se tomaron $100 \mu \mathrm{l}$ se adicionaron en $900 \mu \mathrm{l}$ de agua peptonada bufferada, cada dilución se realizó por triplicado para poder crear el set de NMP, se llevó a incubación por 24 horas a $37^{\circ} \mathrm{C}$ (en las muestras de esponja se hicieron diluciones 1/10 a partir del agua peptonada donde se encontraba la muestra). Después de incubación, se tomaron $1000 \mu \mathrm{l}$ de cada dilución y se transfirieron a tubos que contienen $500 \mu \mathrm{l}$ de agar RappaportVassiliadis semi-sólido y se incubaron por 24 horas a $42^{\circ} \mathrm{C}$. Terminada la incubación los tubos que tuvieron crecimiento y pérdida del color verde se consideraron como un presunto positivo de la presencia de Salmonella y se aislaron en Agar Hektoen por 24 horas a $35^{\circ} \mathrm{C}$, seguido de la incubación las colonias típicas de Salmonella que crecieron fueron purificadas en CHROMagar $^{\mathrm{TM}}$ Salmonella Plus $\mathrm{y}$ confirmadas en el equipo MALDI-TOF MS Bruker Daltonics. Por último, las colonias que fueron confirmadas como Salmonella se tomaron como positivo para el NMP y su lectura se realizó por medio de una tabla para series de tres tubos. La corrección de unidades de $\mathrm{mL} \mathrm{a} \mathrm{cm}^{2}$ de las muestras que fueron tomadas por el método de esponja se realizó tomando en cuenta el área de muestreo el cual fue de $400 \mathrm{~cm}^{2}$ y empleando la ecuación 1. 


\section{RESULTADOS Y DISCUSIÓN}

En el muestreo realizado en la empresa $A$ se obtuvieron en todas las etapas valores menores o cercanos a $3 \mathrm{NMP} / \mathrm{g}$, siendo el corte de pierna en el muestreo 3 la etapa de mayor contaminación con un valor de 4,252 $\mathrm{NMP} / \mathrm{g}$. Esto indica que el proceso de desposte y comercialización de la carne se encuentra bajo control, lo cual obedece a que esta industria cuenta con un sistema HACCP que ha sido reconocido por el INVIMA, el cual promueve su implementación según el decreto 60 de 2002 en las fábricas de alimentos. Además el control de la temperatura (por debajo de $12^{\circ} \mathrm{C}$ ) en esta planta, es un factor importante que afecta la concentración de Salmonella, ya que previene la colonización de la bacteria en la carne por largos periodos, además de realizar una limpieza y desinfección adecuada al finalizar las jornadas de trabajo (Berends et al, 1998 ${ }^{b}$.

En un estudio realizo por Predergast et al, (2008) determinaron la concentración de Salmonella spp. en cortes de cerdo por la técnica de NMP, donde obtuvieron como resultados valores $<3 \mathrm{NMP} / \mathrm{g}$ de las muestras procesadas, datos similares a los obtenidos en la empresa A. También observaron que en algunas ocasiones se encontraba una mayor contaminación de Salmonella en las muestras de la tarde en comparación de las que tomaban en la mañana, lo que concuerda con lo reportado por Berends et al (1998 $)$ quien dice que los efectos de limpieza y desinfección son efectivos durante las primeras horas de producción, pero al pasar el tiempo los efectos disminuyen por el constante paso de canales positivos para Salmonella.

La etapa con mayor concentración de Salmonella spp. en el muestreo realizado en la empresa $B$ fue en la etapa de comercialización en el muestreo 5 con una concentración de 149,148 NMP/g seguido de Iomo con 51,254 NMP/g en el muestreo 3 y 33, $396 \mathrm{NMP} / \mathrm{g}$ en el muestreo 5. La contaminación de la carne de cerdo con Salmonella spp. se puede atribuir a diferentes fuentes (Rodríguez, 2014), dentro de las que se incluyen: cerdos infectados desde la granja y contaminación cruzada durante el sacrificio, aspectos que juegan un papel importante en la contaminación final en mayor o menor medida de la canal, lo cual 
puede afirmar que los niveles altos de concentración reportados en la etapa de comercialización en la empresa B se debe a que la canal entró a planta con un nivel significativo de concentración de Salmonella spp. (Wong et al, 2002). Esta situación la afirma Arguello et al (2013), en su estudio donde identificaron que los serotipos de Salmonella aislados en corrales de reposo presentaban el mismo perfil a lo largo del resto de la cadena de producción, lo que representa un riesgo para la salud humana ya que la planta de desposte y los puntos de comercialización son las últimas etapas de procesamiento antes de llegar la carne al consumidor.

Por último se comparó el comportamiento de las dos empresas donde se evidencia que las concentraciones de Salmonella son inferiores en la empresa $A$ respecto a las obtenidas en la empresa B. Esto pudo ser influenciado por los diversos factores explicados anteriormente. Cada empresa tiene y maneja sus propios protocolos de control y limpieza, por lo que, el mapeo microbiológico deja evidenciar si las medidas tomadas por estas tienen un efecto en la disminución o control del patógeno. Sin embargo es importante que cumplan las Buenas Prácticas de Manufactura, se recomienda la implementación sistemas de aseguramiento de inocuidad como HACCP; y además llevar a cabo en los sitios de comercialización un monitoreo regular de las temperaturas de almacenamiento del producto (Berends et al 1998; ; Wong et al, 2002).

\section{CONCLUSIONES}

La etapa que se determinó con una mayor concentración de Salmonella spp. en la empresa $A$ fue el corte de pierna en el muestreo 3 en la etapa de comercialización con un valor de 4,252 NMP/g. La implementación de sistemas de seguridad como HACCP ayudan a reducir y mantener bajo control la presencia de patógenos en la industria, como se pudo observar en esta empresa, la cual mostro mejores condiciones a lo largo de las etapas.
En la empresa B la etapa de mayor concentración de Salmonella spp. fue la etapa de comercialización con una concentración de $149,148 \mathrm{NMP} / \mathrm{g}$ en el muestreo 5, siendo los principales factores de contaminación en estas etapas, cerdos contaminados desde granja, contaminación cruzada durante el proceso y perdida de cadena de frío. 


\section{REFERENCIAS BIBLIOGRAFICAS}

Arguello H, Carvajal A, Naharro G, Arcos M, Rodicio M, Martin C, et al., Sero- and genotyping of Salmonella in slaughter pigs, from farm to cutting plant, with a focus on the slaughter process. (2013). International Journal of Food Microbiology. 161(1): 4452.

Disponible

en:

https://doi.org/10.1016/j.ijfoodmicro.2012.1 1.013.

aBerends BR, Van Knapen F, Mossel DAA, Burt SA, Snijders JMA. Impact on human health of Salmonella spp. on pork in The Netherlands and the anticipated effects of some currently proposed control strategies. (1998). International Journal of Food Microbiology. 44(3):219-229. Disponible en: $\quad$ https://doi.org/10.1016/S01681605(98)00121-4

bBerends BR, Van Knapen F, Mossel DAA, Burt SA, Snijders JMA. Salmonella spp. On pork at cutting plants and at the retail level and the influence of particular risk factors. (1998). International Journal of Food Microbiology. 44(3):207-217. Disponible en: $\quad$ https://doi.org/10.1016/S0168$\underline{1605(98) 00122-6}$

Berends BR, Urlings HAP, Snijders JMA, Van Knapen F. Identification and quantification of risk factors in animal management and transport regarding Salmonella spp. in pigs. (1996). International Journal of Food Microbiology. 30(1):37-53.

Guerrero J. (2016). Protocolo de Vigilancia en Salud Pública. Enfermedades Transmitidas por alimentos y aguas. Disponible en: http://www.hosusana.gov.co/sites/default/fi les/u1/capacitacion/PRO\%20Enfermedade s\%20Trans.\%20por\%20alimentos.pdf

Instituto Nacional de Salud. Perfil de riesgo Salmonella spp. (no tifoidea) en pollo entero y en piezas. (2011). Revista Cultura Científica. Disponible en: https://www.minsalud.gov.co/sites/rid/Lists/ BibliotecaDigital/RIDE/IA/INS/perfilsalmonella-spp.pdf

Maldonado, Natalie. (2016). Incidencia de posibles bacterias causantes de mastitis en los municipios de Pamplona, Pamplonita y Toledo, Norte de Santander. Revista @limentech, Ciencia y Tecnología Alimentaria. ISSN 1692-7125. Volumen 14, $N^{\circ} 1$, p. $17-26$.

Organización Mundial de la Salud. (2017). Salmonella (no tifoidea). Disponible en: 
http://www.who.int/mediacentre/factsheets/ fs139/es/

Organización Mundial de la Salud. (2015). Las enfermedades de transmisión alimentaria (ETA) en la Región de las Américas de la OMS. Carga mundial de enfermedades de transmisión alimentarias: estimaciones de la OMS. Disponible en: http://www.who.int/foodsafety/areas work/f oodborne-diseases/amro es.pdf?ua $=1$

Pavic A, Groves P, Bailey G, Cox, JM. A validated miniaturized MPN method, based on ISO $6579: 2002$, for the enumeration of Salmonella from poultry matrices. (2010). Journal of Applied Microbiology. 109(1):2534.

Parra M, Durango J, Máttar S. (2002). Microbiología, patogénesis, epidemiología, clínica y diagnóstico de las infecciones producidas por Salmonella. Revista MVZ Córdoba. 7(2):187-200. Disponible en: http://www.redalyc.org/articulo.oa?id=6937 $\underline{0201}$

Prendergast D. Duggan S. Fanning S. Cormican M. Gonzales-Barron U. Butler F. Duffy G. (2008). Prevalence and numbers of Salmonella spp. and Enterobacteriaceae on pork cuts in abattoirs in the Republic of Ireland. Journal of Applied Microbiology. 157(1):82-87. Disponible en: https://doi- org.ezproxy.javeriana.edu.co/10.1111/j.13 65-2672.2008.03854.x

Porkcolombia. Informe económico del mes de enero 2018.

Pua, R. Amparo L. y Navas, N. M. G. (2014). Calidad higiénica y determinación de escherichia coli y salmonella spp en carne de cerdo en expendios de barranquilla. Revista @limentech, Ciencia y Tecnología Alimentaria. ISSN 1692-7125. Volumen 12, $N^{\circ} 1$, p. $15-22$.

Rodríguez D, Suárez M. (2014). Salmonella spp. in the pork supply chain: a risk approach. Revista Colombiana de Ciencias Pecuarias. 27(2):65-75. Disponible en: http://www.redalyc.org/articulo.oa?id=2950 30559002

Rodríguez D. Carrascal AK. Manual toma de muestras de Salmonella spp y Escherichia coli biotipo I en canales porcinas.

Wong L. Hald T. van der Wolf P. Swanenburg M. (2002). Epidemiology and control measure for Salmonella in pigs and pork. Livestock Production Science. 76(3):215222. Disponible en: https://doi.org/10.1016/S03016226(02)00121-5 